\title{
Repetitive Transcranial Magnetic Stimulation Enhances BDNF-TrkB Signaling in Both Brain and Lymphocyte
}

\author{
Hoau-Yan Wang, ${ }^{1}$ Domenica Crupi, ${ }^{1,2}$ JingJing Liu, ${ }^{1}$ Andres Stucky, ${ }^{1}$ Giuseppe Cruciata, ${ }^{1}$ Alessandro Di Rocco, ${ }^{3}$ \\ Eitan Friedman, ${ }^{1}$ Angelo Quartarone, ${ }^{1,2,3}$ and M. Felice Ghilardi ${ }^{1,3}$ \\ ${ }^{1}$ Department of Physiology, Pharmacology \& Neuroscience, Sophie Davis School of Biomedical Education, The City University of New York Medical School, \\ New York, New York 10031, 2Department of Neurological, Psychiatric and Anesthesiological Sciences, University of Messina, Messina 98100, Italy, and \\ ${ }^{3}$ Department of Neurology, New York University, New York, New York 10016
}

Repetitive transcranial magnetic stimulation (rTMS) induces neuronal long-term potentiation or depression. Although brain-derived neurotrophic factor (BDNF) and its cognate tyrosine receptor kinase B (TrkB) contribute to the effects of rTMS, their precise role and underlying mechanism remain poorly understood. Here we show that daily $5 \mathrm{~Hz}$ rTMS for 5 d improves BDNF-TrkB signaling in rats by increasing the affinity of BDNF for TrkB, which results in higher tyrosine-phosphorylated TrkB, increased recruitment of PLC- $\gamma 1$ and shc/N-shc to TrkB, and heightened downstream ERK2 and PI-3K activities in prefrontal cortex and in lymphocytes. The elevated BDNFTrkB signaling is accompanied by an increased association between the activated TrkB and NMDA receptor (NMDAR). In normal human subjects, $5 \mathrm{~d}$ rTMS to motor cortex decreased resting motor threshold, which correlates with heightened BDNF-TrkB signaling and intensified TrkB-NMDAR association in lymphocytes. These findings suggest that rTMS to cortex facilitates BDNF-TrkB-NMDAR functioning in both cortex and lymphocytes.

\section{Introduction}

Repetitive transcranial magnetic stimulation (rTMS) is a noninvasive brain-stimulation procedure noted for its effects on emotional, cognitive, sensory, and motor functions in patients with neuropsychiatric diseases (Rossi et al., 2009). Indeed, multiple rTMS sessions are used to treat depression, parkinsonian motor signs, writer's cramp, tinnitus, and aphasia (Fregni and PascualLeone, 2007; Elahi et al., 2009; Vedeniapin et al., 2010). Despite the reported beneficial effects, the biochemical mechanisms of rTMS action are far from clear. It is likely that rTMS induces long-term potentiation (LTP) or depression, which, in turn, produce lasting changes on neocortical excitability and synaptic connections (Esser et al., 2006; Quartarone et al., 2006; Di Lazzaro et al., 2010). In humans, LTP-like phenomena following $5 \mathrm{~Hz}$ rTMS have been documented by increases in motor-evoked potential (MEP) amplitude (Quartarone et al., 2006; Conte et al., 2008), regional cerebral blood flow, glucose metabolism (Siebner et al., 2000, 2001), and EEG response amplitude (Esser et al., 2006). Studies in animals have shown that rTMS effects depend on

Received April 28, 2011; revised June 2, 2011; accepted June 10, 2011.

Author contributions: H.-Y.W., A.D.R., E.F., A.Q., and M.F.G. designed research; H.-Y.W., D.C., J.L., A.S., and G.C. performed research; H.-Y.W., D.C., J.L., A.S., G.C., and M.F.G. analyzed data; H.-Y.W., A.D.R., E.F., A.Q., and M.F.G. wrote the paper.

This research was supported by the National Parkinson's Foundation (M.F.G., A.D.R.), NIH-NINDS Grant R01054864 (M.F.G.), NIH-NIDA Grant R24-DA018055 (E.F.), and the Mac Lipkin Fellowship (G.C.). We also thank Dr. A. Naro for technical assistance.

The authors declare no competing financial interests.

Correspondence should be addressed to Dr. Hoau-Yan Wang, Department of Physiology, Pharmacology \& Neuroscience, Sophie Davis School of Biomedical Education, The City University of New York Medical School, H-210F, Harris Hall, 160 Convent Avenue, New York, NY 10031. E-mail: hywang@sci.ccny.cuny.edu.

DOI:10.1523/JNEUROSCI.2125-11.2011

Copyright $\odot 2011$ the authors $\quad 0270-6474 / 11 / 3111044-11 \$ 15.00 / 0$ changes in NMDA receptor (NMDAR) activity (Wang et al., 2010), the most-recognized mediator of LTP.

In recent years, brain-derived neurotrophic factor (BDNF) and its cognate receptor tyrosine receptor kinase $\mathrm{B}(\operatorname{TrkB})$, a member of the neurotrophin receptor tyrosine kinase family, have emerged as important upstream regulators of LTP in brain regions, including hippocampus and neocortex (Minichiello, 2009; Fritsch et al., 2010). Interestingly, neurotrophin receptors are important for the development of other organs and are present in the kidney, prostate (Pflug et al., 1995), bone marrow derived-endothelial precursor cells (Kermani et al., 2005), heart (Hiltunen et al., 1996), ovaries (Dissen et al., 1995), fibroblasts (Easton et al., 1999), and seminiferous epithelium (Schultz et al., 2001). Moreover, TrkBs are also expressed in structures with immunological functions such as the thymus and T- and B-lymphocytes (Schuhmann et al., 2005; Berzi et al., 2008; De Santi et al., 2009), where they appear to play an important role in cell development and survival (Maroder et al., 1996; Schuhmann et al., 2005). Upon BDNF binding, TrkB is activated by tyrosine phosphorylation through its intrinsic tyrosine kinase, which in turn, enhances downstream ERK2 and PI3K activities, promotes early gene expression, and produces pleiotropic effects that depend on the cellular environment (Longo et al., 2007; Greenberg et al., 2009). Importantly, it remains unclear whether TrkB activation in the CNS is correlated with TrkB activation in peripheral tissues.

Therefore, we first, investigated whether $5 \mathrm{~d}$ rTMS treatment affects BDNF-TrkB signaling and TrkB-NMDAR interaction in prefrontal cortex (PFCX), hippocampus, and lymphocytes of adult rats. Then, we determined whether in human subjects, $5 \mathrm{~d} 5$ $\mathrm{Hz}$ rTMS induced changes in both electrophysiological markers of LTP-like phenomena and BDNF-induced TrkB activation in 
Table 1. Results of ANOVA for BDNF-TrkB signaling and TrkB-NMDAR linkage comparing sham- and rTMS-treated rats

\begin{tabular}{|c|c|c|c|c|c|c|}
\hline & \multicolumn{2}{|c|}{ NMDA/Gly } & \multicolumn{2}{|l|}{$\mathrm{K}^{+}$} & \multicolumn{2}{|l|}{ hBDNF } \\
\hline & $F_{(1,10)}$ & $p$ & $F_{(1,10)}$ & $p$ & $F_{(1,10)}$ & $p$ \\
\hline \multicolumn{7}{|l|}{ PFCX } \\
\hline pY-TRKB (145 kDa) & 10.660 & 0.0085 & 27.810 & 0.0004 & 7.590 & 0.0200 \\
\hline pY-TRKB (95 kDa) & 1.540 & 0.2400 & 1.610 & 0.2300 & 1.690 & 0.2200 \\
\hline PLC $-\gamma 1$ & 10.930 & 0.0079 & 14.720 & 0.0033 & 30.772 & 0.0002 \\
\hline Sch & 35.612 & 0.0001 & 14.224 & 0.0037 & 20.229 & 0.0011 \\
\hline NR1 & 35.490 & 0.0001 & 23.990 & 0.0006 & 27.370 & 0.0004 \\
\hline pY-ERK2 & 11.210 & 0.0074 & 12.770 & 0.0051 & 24.240 & 0.0006 \\
\hline pS-AKT & 32.240 & 0.0002 & 68.990 & $<0.0001$ & 14.190 & 0.0037 \\
\hline N-Shc & 72.000 & $<0.0001$ & 45.990 & $<0.0001$ & 20.220 & 0.0011 \\
\hline NR2A & 27.560 & 0.0004 & 27.670 & 0.0004 & 14.070 & 0.0038 \\
\hline PSD-95 & 23.840 & 0.0006 & 29.161 & 0.0003 & 21.430 & 0.0009 \\
\hline \multicolumn{7}{|l|}{ Hippocampus } \\
\hline pY-TRKB (145 kDa) & 0.360 & 0.5600 & 0.055 & 0.8200 & 0.360 & 0.5600 \\
\hline pY-TRKB (95 kDa) & 0.220 & 0.6500 & 0.430 & 0.5300 & 0.105 & 0.7500 \\
\hline PLC- $\gamma 1$ & 0.004 & 0.9500 & 0.000 & 0.9900 & 0.004 & 0.9500 \\
\hline Sch & 1.040 & 0.3300 & 0.360 & 0.5600 & 0.950 & 0.3500 \\
\hline NR1 & 0.120 & 0.7400 & 0.018 & 0.9000 & 0.046 & 0.8300 \\
\hline pY-ERK2 & 0.710 & 0.4200 & 6.470 & 0.0290 & 0.009 & 0.9300 \\
\hline pS-AKT & 0.024 & 0.8800 & 0.160 & 0.7000 & 5.030 & 0.0490 \\
\hline N-Shc & 0.087 & 0.7700 & 0.110 & 0.7500 & 4.660 & 0.0563 \\
\hline NR2A & 0.350 & 0.5600 & 0.910 & 0.3600 & 0.030 & 0.9600 \\
\hline PSD-95 & 0.230 & 0.6400 & 0.130 & 0.7300 & 0.210 & 0.6600 \\
\hline \multicolumn{7}{|l|}{ Lymphocytes } \\
\hline pY-TRKB (145 kDa) & - & - & - & - & 29.231 & 0.0003 \\
\hline pY-TRKB (95 kDa) & - & - & - & - & 2.772 & 0.1269 \\
\hline PLC- $\gamma 1$ & - & - & - & - & 25.597 & 0.0005 \\
\hline Sch & - & - & - & - & 6.866 & 0.0256 \\
\hline NR1 & - & - & - & - & 12.311 & 0.0056 \\
\hline pY-ERK2 & - & - & - & - & 71.514 & $<0.0001$ \\
\hline pS-AKT & - & - & - & - & 10.691 & 0.0084 \\
\hline
\end{tabular}

Significant results are reported in bold characters.

lymphocytes. We found that, in rats, rTMS augments BDNFinduced TrkB activation in both PFCX and lymphocytes, and these changes are significantly correlated. In humans, rTMS decreases the resting motor threshold (RMT) and increases BDNFinduced TrkB activation in lymphocytes. This is the first direct evidence that rTMS induces changes in the brain BDNF-TrkB signaling that are reflected in lymphocytes.

\section{Materials and Methods}

Animals and treatment protocols

Twelve 10-week-old male Sprague Dawley rats from Taconic Farm were housed individually in a $12 \mathrm{~h}$ light/dark cycle with food and water ad libitum. After adaptation, the animals were observed for 2 weeks to verify their circadian rhythm and then handled daily for a week with a rodent coil placed over their head to simulate rTMS. Six animals underwent five rTMS sessions daily for 5 consecutive days (rTMS group), while six animals served as controls (sham group).

In the rTMS group, a rodent coil (Magstim, custom-made) was placed directly touching the skull of animal that was held gently in a flexible plastic rat restrainer. A Magstim Rapid Rate Stimulator delivered 1600 stimuli at $5 \mathrm{~Hz}$, at $50 \%$ of maximum output in four blocks of 400 each, in $2.5 \mathrm{~min}$ with $1 \mathrm{~min}$ interblock interval. In the sham group, stimuli were delivered with the coil rotated $90^{\circ}$ about the axis of the handle and separated from the head using a $2 \mathrm{~cm}$ plastic spacer cube. This ensured that the animal felt the vibrations produced by the click of the TMS coil without brain stimulation (Esser et al., 2006). No changes in either spontaneous behavior or circadian rhythms were observed during the entire period of either treatment. The day after last treatment, all animals were killed at 10:00-11:00 A.M. to minimize the intersubject BDNF variability. BDNF levels are uniformly low after a few sleep cycles but vary highly between animals during the day, depending upon their activity (Lu et al., 2008). The animals were then sedated with $5 \%$ halothane inhalation, placed in a stereotaxic equipment with the head positioned $45^{\circ}$ down- ward for CSF sampling, and then decapitated. The brain tissue was processed for biochemical analysis and trunk blood was collected to isolate serum for BDNF/pro-BDNF levels and lymphocytes for BDNF-induced TrkB signaling and TrkB-NMDA receptor association determinations.

All animal procedures complied with the National Institutes of Health Guide for Care and Use of Laboratory Animals. The experimental protocol was approved by the City College of New York Animal Care and Use Committee.

\section{Materials and chemicals}

Recombinant human BDNF (rhBDNF), leupeptin, aprotinin, phenylmethylsulfonyl fluoride (PMSF), pepstatin A, soybean trypsin inhibitor, $\mathrm{NaF}$, sodium vanadate, $\beta$-glycerophosphate, 2 -mercaptoethanol, NMDA, glycine, Tween 20, NP-40, and Histopaque-1077 were from Sigma. Anti-PSD-95 (05494) was from Millipore. Anti-TrkB (SC-8316), -pY-Trk (SC-8058), -BDNF/pro-BDNF (SC-2098), -NT3 (SC-547), -NT4 (SC-545), -phosphotyrosine (SC-508), -ERK2 (SC-154, SC81457), -pY-ERK (SC-7383), -pS ${ }^{473}$ Akt (SC-7985-R), -Akt1 (SC65487), -Akt2 (SC-81436), -Akt1/2/3 (SC-8312), -phospholipase C- $\gamma 1$ (SC-7290), -NR1 (SC-9058), -NR2A (SC-9056), -NR2B (SC-9057), -actin (SC-7210), and - $\beta$-actin (SC-47778) were from Santa Cruz Biotechnology. Seize-X immunoprecipitation kit, antigen elution buffer, Bind NeutrAvidin, high binding capacity coated 96-well plates, and West Pico chemiluminescent reagents were from Pierce-Endogen. Bradford reagent, SDS-PAGE reagents, and prestained molecular weight markers were from Bio-Rad. Protease inhibitors (EDTA-free) and protein phosphatase inhibitor tablets were from Roche.

BDNF was reconstituted according to the manufacturer's instruction. To avoid freezing damages, $10 \%$ glycerol was added to achieve $10 \mathrm{ng} / \mu \mathrm{l}$ BDNF and stored in $-80^{\circ} \mathrm{C}$ until use. All other test agents were made freshly according to the manufacturer's recommendation. The DMSO concentration in the incubation medium was $\leq 1 \%$ when used.

Brain slice preparation. PFCX and hippocampi were sliced using a chilled Mcllwain tissue chopper $(100 \mu \mathrm{m} \times 100 \mu \mathrm{m} \times 3 \mathrm{~mm})$. Approx- 
imately $10 \mathrm{mg}$ brain slices were suspended in 1 $\mathrm{ml}$ of ice-cold oxygenated low- $\mathrm{Mg}^{2+}$ Krebs'Ringer (LMKR), containing 25 mM HEPES, $\mathrm{pH}$ 7.4, $118 \mathrm{~mm} \mathrm{NaCl}, 4.8 \mathrm{~mm} \mathrm{KCl}, 1.3 \mathrm{~mm} \mathrm{CaCl}_{2}$, $1.2 \mathrm{~mm} \mathrm{KH}_{2} \mathrm{PO}_{4}, 0.3 \mathrm{~mm} \mathrm{MgSO}_{4}, 25 \mathrm{~mm}$ $\mathrm{NaHCO}_{3}, 10 \mathrm{~mm}$ glucose, $100 \mu \mathrm{M}$ ascorbic acid, $50 \mu \mathrm{g} / \mathrm{ml}$ leupeptin, $0.2 \mathrm{~mm}$ PMSF, 25 $\mu \mathrm{g} / \mathrm{ml}$ pepstatin $\mathrm{A}$, and $0.01 \mathrm{U} / \mathrm{ml}$ soybean trypsin inhibitor and centrifuged briefly. Brain slices were washed twice more and suspended in $1 \mathrm{ml}$ of LMKR.

CSF collection and preparation. Anesthetized rats were placed in a stereotaxic apparatus with the head pointing $45^{\circ}$ downward. A syringeconnected needle was inserted horizontally and centrally into the cisterna magna, and colorless CSF was slowly aspirated into a syringe (Nirogi et al., 2009). Protein concentration was measured by the Bradford method; BDNF was determined by Western blotting.

Lymphocyte preparation. To prepare lymphocytes, $1 \mathrm{ml}$ of rat trunk blood was collected into a $1.5 \mathrm{ml}$ Eppendorf centrifuge tube containing $1.0 \mathrm{~mm}$ EDTA. Collected blood $(0.9 \mathrm{ml})$ was layered onto $0.9 \mathrm{ml}$ of Histopaque-1077 at $25^{\circ} \mathrm{C}$, and the entire content was centrifuged at $400 \times g$ for $30 \mathrm{~min}\left(25^{\circ} \mathrm{C}\right)$ to yield plasma (top layer) and lymphocytes (opaque interface). The lymphocytes were washed twice by mixing with $3 \mathrm{ml}$ of LMKR followed by centrifugation at $250 \mathrm{~g}$ for $10 \mathrm{~min}$ and resuspension. The final pellet was resuspended in $250 \mu \mathrm{l}$ of LMKR before protein content determination by the Bradford method and assessment of BDNFTrkB signaling.

Ex vivo tissue treatment and assessments of $B D N F-T r k B$ signaling and TrkB-NMDAR association. To determine the effect of rTMS on TrkB signaling, brain slices were incubated for $30 \mathrm{~min}$ at $37^{\circ} \mathrm{C}$ in $0.5 \mathrm{ml}$ of LMKR without or with $50 \mathrm{ng} / \mathrm{ml} \mathrm{rhBDNF}, 10 \mu \mathrm{M}$ NMDA/ $1 \mu \mathrm{M}$ glycine, or $20 \mathrm{~mm} \mathrm{~K}^{+}$. The incubation mixture was aerated with $95 \% \mathrm{O}_{2} / 5 \% \mathrm{CO}_{2}$ every $10 \mathrm{~min}$ for $1 \mathrm{~min}$ during the incubation.

To determine rTMS effect on lymphocyte TrkB signaling, lymphocytes $(200 \mu \mathrm{g})$ were incubated for $30 \mathrm{~min}$ at $37^{\circ} \mathrm{C}$ in $0.5 \mathrm{ml}$ of LMKR without or with $50 \mathrm{ng} / \mathrm{ml} \mathrm{rhBDNF}$. The incubation mixture was aerated with $95 \% \mathrm{O}_{2} / 5 \% \mathrm{CO}_{2}$ every $10 \mathrm{~min}$ for $1 \mathrm{~min}$ during the incubation.

Ligand stimulation was terminated by adding $1 \mathrm{ml}$ of ice-cold $\mathrm{Ca}^{2+}$-free LMKR containing $0.5 \mathrm{~mm}$ EGTA/0.1 $\mathrm{mm}$ EDTA and phosphatase inhibitors followed by centrifugation. The resultant brain slices or lymphocytes were homogenized in $0.25 \mathrm{ml}$ of ice-cold immunoprecipitation buffer. The homogenates were centrifuged at $1000 \times g$ for $5 \mathrm{~min}$ $\left(4^{\circ} \mathrm{C}\right)$, and the supernatant (postmitochondrial fraction) was sonicated for $10 \mathrm{~s}$ on ice and solubilized in $0.5 \%$ digitonin $/ 0.2 \%$ sodium cholate $/ 0.5 \%$ $\mathrm{NP}-40$ for $60 \mathrm{~min}\left(4^{\circ} \mathrm{C}\right)$ with end-to-end rotation. The resultant lysates were cleared by centrifugation at $50,000 \times g$ for $5 \mathrm{~min}$ and diluted with $0.75 \mathrm{ml}$ of immunoprecipitation buffer and protein concentrations measured by the Bradford method.

Assessment of TrkB activation; phospholipase C- $\gamma 1$, shc, and N-shc recruitment; TrkB-NMDAR interaction; and ERK and PI3K activation by coimmunoprecipitation

TrkB signaling complexes, ERK2, and Akt in $200 \mu \mathrm{g}$ of tissue lysate were immunoprecipitated separately by a $2 \mathrm{~h}$ incubation $\left(4^{\circ} \mathrm{C}\right)$ with $1 \mu \mathrm{g}$ of immobilized anti-TrkB (for assessment of pY-TrkB; PLC- $\gamma 1$, shc, and $\mathrm{N}$-shc recruitment; and TrkB-NMDAR interaction), anti-ERK2 (pYERK2), and mouse anti-Akt1/2 (pS ${ }^{473}$-Akt) followed by addition of 25 $\mu \mathrm{l}$ of protein $\mathrm{A} / \mathrm{G}$-conjugated agarose beads, and incubation continued at $4^{\circ} \mathrm{C}$ for $16 \mathrm{~h}$. The resultant immunocomplexes were pelleted by centrifugation $\left(4^{\circ} \mathrm{C}\right)$, washed three times with $1 \mathrm{ml}$ of ice-cold $\mathrm{PBS}, \mathrm{pH} 7.2$, and centrifuged. The resultant immunocomplexes were solubilized by boiling for 5 min in $100 \mu$ l of SDS-PAGE sample preparation buffer $(62.5$ mм Tris- $\mathrm{HCl}, \mathrm{pH} 6.8,10 \%$ glycerol, 2\% SDS, 5\% 2-mercaptoethanol, and $0.1 \%$ bromophenol blue). The contents of the pY-TrkB, PLC- $\gamma 1$, shc, N-shc, NMDAR subunits, and PSD-95 in 50\% anti-TrkB immunoprecipitate, pY-ERK2 in 50\% anti-ERK2 immunoprecipitate, and $\mathrm{pS}^{473} \mathrm{Akt}$ in $50 \%$ anti-Akt immunoprecipitate were determined by Western blotting. The blots were stripped and reprobed with anti-TrkB, -ERK2, or -Akt1/2/3 to illustrate even immunoprecipitation efficiency and loading.

Western blot analysis. Solubilized immunoprecipitates size-fractionated by either 7.5 or $10 \%$ SDS-PAGE were electrophoretically transferred to nitrocellulose membranes. The membranes were washed with PBS and blocked overnight $\left(4^{\circ} \mathrm{C}\right)$ with $10 \%$ milk in $0.1 \%$ Tween 20 -containing PBS (PBST). Following three 5 min $0.1 \%$ PBST washes, the membranes were incubated at $25^{\circ} \mathrm{C}$ for $2 \mathrm{~h}$ with 1:500 to 1:1,000 dilutions of selected antibodies. After three 2 min $0.1 \%$ PBST washes, membranes were incubated for $1 \mathrm{~h}$ with anti-species IgG-HRP (1:5000 dilution) and washed three times with $0.1 \%$ PBST ( 2 min each). The signals were detected using a chemiluminescent method and visualized by exposure to $\mathrm{x}$-ray film. Specific bands were quantified by densitometric scanning (GS-800 calibrated densitometer, Bio-Rad).

$B D N F$ binding. PFCX synaptosomes of sham- and rTMS-treated rats were the tissue source for determining rTMS effects on BDNF binding characteristics. Membrane-bound proteins in the synaptosomes were first biotinylated using a biotinylation kit. The biotinylated surface proteins were extracted from synaptic membranes using $0.5 \%$ digitonin/ $0.2 \%$ sodium cholate $/ 0.5 \% \mathrm{NP}-40$. Following $1: 5$ dilution, the protein concentrations were measured using the Bradford method. To coat the plate with biotinylated proteins, streptavidin-coated plates (Reacti-Bind NeutrAvidin high binding capacity 96-well plate) were washed three 
times with $200 \mu \mathrm{l}$ of ice-cold LMKR and then incubated at $30^{\circ} \mathrm{C}$ with 50 $\mu \mathrm{g} /$ well biotinylated receptor solution for $1 \mathrm{~h}$ in the presence of $5 \%$ blocking reagent (Thermo). Following two LMKR washes, BDNF (100 fM to $10 \mathrm{~nm}$ ) was added, and incubation was performed at $30^{\circ} \mathrm{C}$ for $1 \mathrm{~h}$. The plate was washed with ice-cold LMKR and incubated at $30^{\circ} \mathrm{C}$ for $1 \mathrm{~h}$ with anti-BDNF $(0.5 \mu \mathrm{g} /$ well $)$, followed by FITC-conjugated anti-rabbit IgG $(0.5 \mu \mathrm{g} /$ well $)$ for $1 \mathrm{~h}$. Plates were washed twice with $200 \mu \mathrm{l}$ of ice-cold LMKR, and the FITC signals were determined by a multimode plate reader, DTX880 (Beckman). Negligible FITC signal was noted when vehicle instead of BDNF was added.

\section{Human subjects and treatment protocols}

The experiments were conducted on eight healthy right-handed subjects, without neuropsychiatric disorder ( 7 men, age range: $21-32$ years; mean age: 26 years). All subjects underwent a double blind, placebo-controlled, crossover study where they received active or sham stimulation daily for 5 consecutive days. Stimuli were delivered through a repetitive biphasic magnetic stimulator (Magstim Super-Rapid). Two eight-shaped coils (outer diameters: $9 \mathrm{~cm}$ ) were stacked over the motor hot spot of the first dorsal interosseus muscle (FDI) with a $45^{\circ}$ orientation, perpendicularly to the central sulcus. For "active stimulation," the coil directly contacting the scalp was connected to the stimulator, whereas the other was not. For "sham stimulation," the reverse was true. Hence, the subjects have similar perception of coil/scalp contact and discharge noise. The rTMS intensity was set at $90 \%$ RMT. In both active and sham sessions, participants received four 300-pulse rTMS trains (1200 stimuli) with $1 \mathrm{~min}$ between-session interval daily for 5 consecutive days. Active and sham rTMS sessions were given in a counterbalanced order a week apart.

RMT in the FDI was measured before each daily rTMS sessions and at the end of the whole study according to international guidelines (Rossini et al., 1994). After each RMT measurement, a suprathreshold singlepulse TMS at an intensity of $125 \%$ of RMT every $5 \mathrm{~s}$ was delivered to collect 20 consecutive MEPs from the right FDI at rest. Peak-to-peak amplitude of each MEP was measured off-line, and the mean MEP amplitude was calculated. MEPs were recorded from $\mathrm{Ag}-\mathrm{AgCl}$ surface electrodes over the right FDI using a belly-tendon montage. The signal was amplified and bandpass filtered ( $32 \mathrm{~Hz}$ to $1 \mathrm{kHz}$ ) by a DIGITIMER D150 amplifier (Digitimer) and stored at a sampling rate of $5 \mathrm{kHz}$ on a personal computer for off-line analysis (SigAvg Software, Cambridge Electronic Design). EMG activity of the right FDI muscle was continuously monitored with visual (oscilloscope) and auditory (speakers) feedback during the entire experiment. For MEPs evoked by single transcranial stimuli peak-to-peak amplitudes were measured and averaged for each experimental condition, using NuCursor software (Sobell Research Department of Motor Neuroscience and Movement Disorders, Institute of Neurology, University College of London). No subject experienced adverse effects of the rTMS and sham procedures or was able to differentiate between rTMS and sham sessions.

To measure the rTMS effects on BDNF-TrkB signaling in lymphocytes, three blood samples were collected: first day, before the rTMS session; same day immediately after the rTMS session; and the fifth day, after the last stimulation.

All subjects gave written informed consent before the study. Institutional ethics committees approved the experiments.

\section{Statistical analyses}

All data are presented as mean \pm SEM. Treatment effects were evaluated by ANOVA with Greenhouse-Geisser correction for nonsphericity. Specifically, the treatment effects (rTMS vs sham) of the TrkB activationrelated biochemical indices in animal experiments were evaluated using one-way ANOVAs. To analyze both biochemical and electrophysiological data in the human studies, we used mixed model ANOVA with treatment (rTMS vs sham) as a between-subject factor and time (before day 1, after day 1, after day 5) as within-subject factor. Post hoc tests were then used with Bonferroni corrections for multiple comparisons.

The threshold for significance was $p<0.05$. Between-group comparisons were also conducted for all parameters.
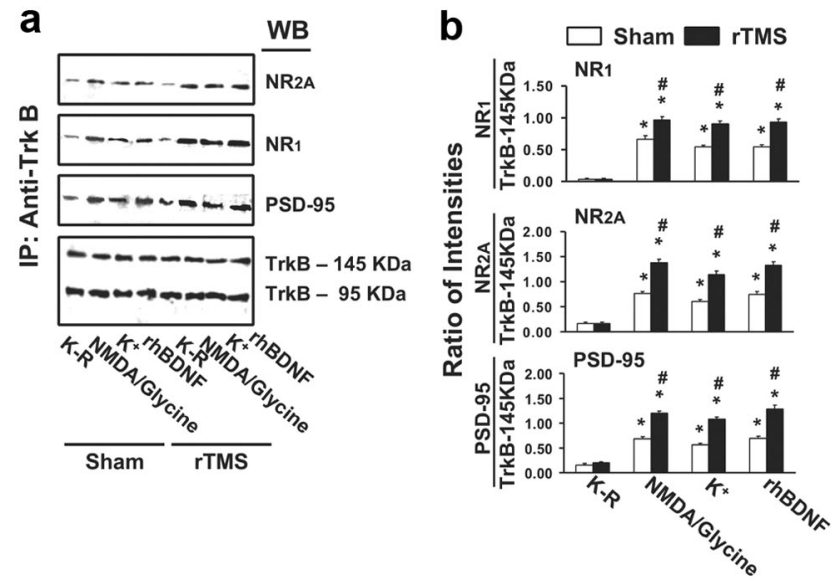

Figure 2. rTMS treatments increase BDNF-induced association of the NMDARs and NMDAR synaptic anchoring protein PSD-95 with TrkB in prefrontal cortex. $\boldsymbol{a}, \boldsymbol{b}$, A representative blot (a) and normalized densitometric data $(\boldsymbol{b})$ showing the Western analysis of the effect of $5 \mathrm{drTMS}$ on the levels of NMDAR subunits NR1 and NR2A as well as NMDAR synaptic anchoring protein PSD-95 associated with TrkB in response to exogenously added rhBDNF or endogenously release BDNF by $\mathrm{K}^{+}$-depolarization or $10 \mu \mathrm{M}$ NMDA $+1 \mu \mathrm{m}$ glycine in the anti-TrkB immunoprecipitates of prefrontal cortical slice lysates prepared from sham-treated or $5 \mathrm{~d}$ rTMS rats. The blots were stripped and reprobed with anti-TrkB to measure immunoprecipitation efficiency and loading. The data are means \pm SEM of ratio of the optical intensity of the NR1, NR2A, and PSD-95 to the TrkB-145 kDa band derived from six independent determinations. ${ }^{*} p<0.01$ compared to respective $K-R$-treated level in the same group. ${ }^{\#} p<0.01$ compared to respective response in the sham control group.

Table 2. Quantitative analysis of BDNF-TrkB signaling and TrkB-NMDAR linkage in the hippocampus of sham- and rTMS-treated rats

\begin{tabular}{llll}
\hline$K-R$ & $\begin{array}{llll}\text { NMDA/ } \\
\text { glycine }\end{array}$ & $K^{+}$ & rhBDNF \\
\hline
\end{tabular}

SHAM

pY-TrkB $145 \mathrm{kDa} /$ TrkB $145 \mathrm{kDa} \quad 0.13 \pm 0.01 \quad 1.01 \pm 0.07 \quad 1.09 \pm 0.05 \quad 1.28 \pm 0.09$ pY-TrkB 95 kDa/TrkB 95 kDa $\quad 0.15 \pm 0.02 \quad 0.82 \pm 0.06 \quad 0.70 \pm 0.04 \quad 0.89 \pm 0.09$ $\begin{array}{llllll}\mathrm{PLC} \gamma 1 / \text { TrkB } 145 \mathrm{kDa} & 0.03 \pm 0.01 & 0.69 \pm 0.09 & 0.61 \pm 0.08 & 0.81 \pm 0.13\end{array}$ N-Shc/TrkB $145 \mathrm{kDa} \quad 0.14 \pm 0.01 \quad 1.15 \pm 0.11 \quad 1.04 \pm 0.08 \quad 1.34 \pm 0.11$ $\begin{array}{llllll}\mathrm{Shc} / \mathrm{TrkB} 145 \mathrm{kDa} & 0.15 \pm 0.01 & 1.63 \pm 0.16 & 1.46 \pm 0.16 & 1.90 \pm 0.14\end{array}$ $\begin{array}{llllll}\mathrm{NR} 1 / T \mathrm{rkB} 145 \mathrm{kDa} & 0.23 \pm 0.03 & 1.12 \pm 0.06 & 0.96 \pm 0.06 & 1.34 \pm 0.07\end{array}$ $\begin{array}{llllll}\mathrm{NR} 2 \mathrm{~A} / \mathrm{TrkB} 145 \mathrm{kDa} & 0.18 \pm 0.04 & 0.81 \pm 0.03 & 0.62 \pm 0.03 & 0.89 \pm 0.05\end{array}$ $\begin{array}{lllll}\text { PSD-95/TrkB } 145 \mathrm{kDa} & 0.21 \pm 0.03 & 1.19 \pm 0.08 & 1.08 \pm 0.08 & 1.37 \pm 0.07\end{array}$ $\begin{array}{lllll}\text { pY-ERK2/ERK2 } & 0.21 \pm 0.02 & 0.90 \pm 0.05 & 0.92 \pm 0.04 & 0.95 \pm 0.04\end{array}$ $\begin{array}{lllll}\mathrm{pS}^{473} \text {-Akt/Akt } & 0.07 \pm 0.01 & 0.40 \pm 0.04 & 0.35 \pm 0.04 & 0.45 \pm 0.02\end{array}$ rTMS

pY-TrkB $145 \mathrm{kDa} / \mathrm{TrkB} 145 \mathrm{kDa} \quad 0.15 \pm 0.02 \quad 1.07 \pm 0.08 \quad 1.11 \pm 0.06 \quad 1.18 \pm 0.10$ pY-TrkB 95 kDa/TrkB 95 kDa $\quad 0.15 \pm 0.02 \quad 0.86 \pm 0.06 \quad 0.76 \pm 0.05 \quad 0.90 \pm 0.06$ $\begin{array}{llllll}\mathrm{PLC} \gamma 1 / \text { TrkB } 145 \mathrm{kDa} & 0.03 \pm 0.01 & 0.70 \pm 0.08 & 0.60 \pm 0.06 & 0.80 \pm 0.12\end{array}$ $\mathrm{N}-\mathrm{Shc} / \mathrm{TrkB} 145 \mathrm{kDa}$ $\mathrm{Shc} / \mathrm{TrkB} 145 \mathrm{kDa}$

$\mathrm{NR} 1 / \mathrm{TrkB} 145 \mathrm{kDa}$

NR2A/TrkB $145 \mathrm{kDa}$

PSD-95/TrkB $145 \mathrm{kDa}$ PY-ERK2/ERK2 $\mathrm{pS}^{473}$-Akt/Akt $\begin{array}{llll}0.14 \pm 0.02 & 1.06 \pm 0.07 & 0.98 \pm 0.05 & 1.05 \pm 0.06\end{array}$ $0.16 \pm 0.02 \quad 1.68 \pm 0.16 \quad 1.39 \pm 0.13 \quad 1.53 \pm 0.11$ $0.24 \pm 0.05 \quad 1.16 \pm 0.10 \quad 0.98 \pm 0.11 \quad 1.25 \pm 0.10$ $\begin{array}{lllll}0.16 \pm 0.03 & 0.83 \pm 0.04 & 0.68 \pm 0.07 & 0.86 \pm 0.05\end{array}$ $0.27 \pm 0.04 \quad 1.25 \pm 0.09 \quad 1.13 \pm 0.08 \quad 1.28 \pm 0.09$ $0.18 \pm 0.01 \quad 0.85 \pm 0.05 \quad 0.75 \pm 0.04 \quad 0.96 \pm 0.05$ $0.07 \pm 0.02 \quad 0.42 \pm 0.03 \quad 0.41 \pm 0.02 \quad 0.47 \pm 0.03$

\section{Results}

rTMS in rats facilitates TrkB activation and TrkB-NMDAR interaction in PFC, but not in the hippocampus

Detailed statistical analyses performed on the results obtained from PFCX and hippocampi are reported in Table 1.

In PFCX, the levels of the 145 and $95 \mathrm{kDa}$ forms of TrkB were similar in rTMS and sham groups with or without BDNF 


\section{$\square$ Sham $\square$ rTMS}

a

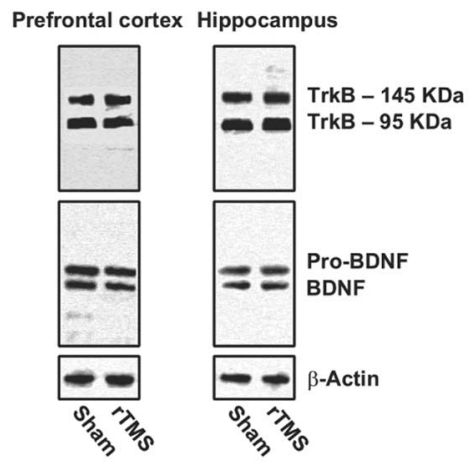

C

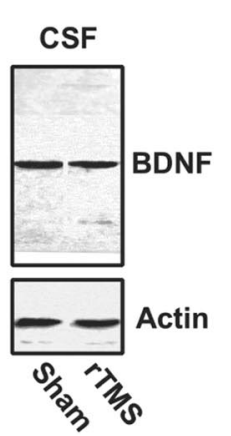

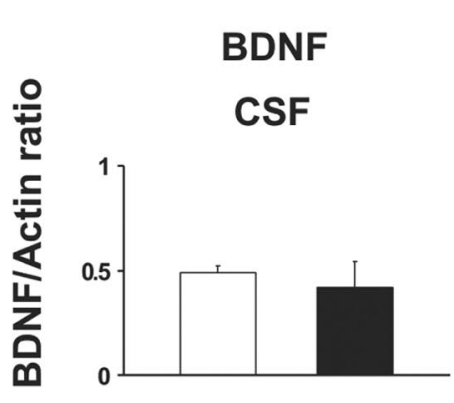

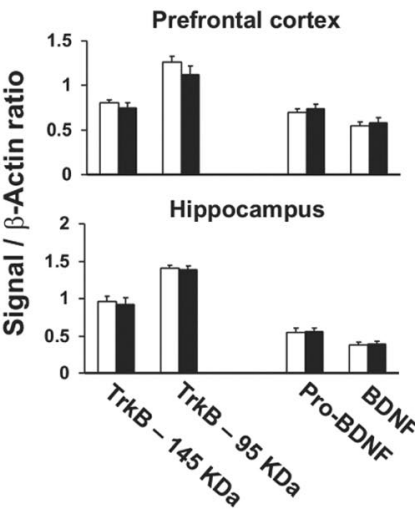

b

Lymphocyte

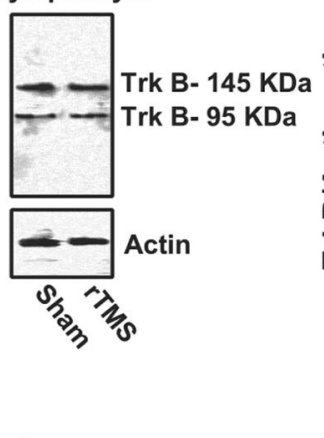

d

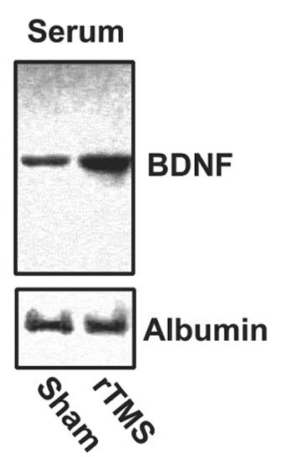

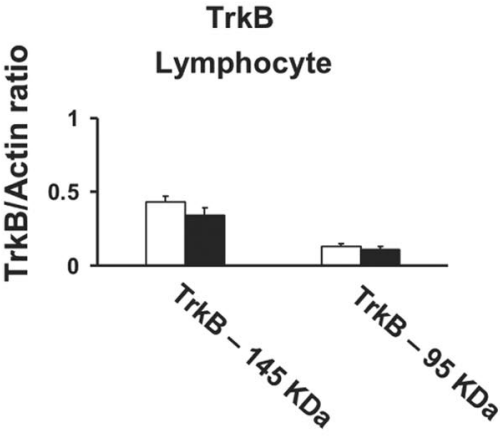

BDNF

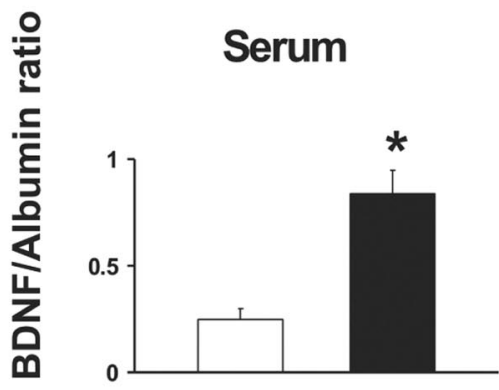

Figure 3. The effect of rTMS treatments on TrkB and pro-BDNF/BDNF expression levels. $\boldsymbol{a}-\boldsymbol{d}$, Representative blots (left) with normalized densitometric data (right) showing the Western analysis of the effect of $5 \mathrm{~d}$ rTMS on the expression levels of 145 and $95 \mathrm{kDa}$ TrkB, pro-BDNF (32 kDa), and BDNF (14 kDa) in PFCX and hippocampus (a); the expression levels of 145 and $95 \mathrm{kDa}$ TrkB in lymphocytes (b); and the expression level of BDNF in CSF (c) and in plasma (d).

exposure (Fig. 1a). BDNF was released from brain slices endogenously by $10 \mu \mathrm{M}$ NMDA/ $1 \mu \mathrm{M}$ glycine (presumably from postsynaptic dendritic fields) or by $20 \mathrm{mM} \mathrm{K}^{+}$-depolarization (presumably from presynaptic axonal terminals) or added exogenously $(50 \mathrm{ng} / \mathrm{ml})$. In slices obtained from sham-treated animals, BDNF significantly increased tyrosine-phosphorylated full-length TrkB (pY-145 kDa TrkB) and truncated TrkB (pY-95 $\mathrm{kDa}$ TrkB). In the rTMS group, the level of BDNF-induced pY$145 \mathrm{kDa}$ TrkB was significantly higher than in the sham group. There were no significant differences between the two groups for the level of pY-95 kDa TrkB (Fig. 1a). The finding of BDNFinduced TrkB phosphorylation/activation in the rTMS group was substantiated by the higher levels of Shc, N-Shc, and PLC- $\gamma 1$ recruited to $\mathrm{pY}$-TrkB (average increases: 60\%) (Fig. 1b). A similar conclusion is also supported by the activation of the downstream signaling cascades of TrkB. In fact, BDNFinduced activation of ERK2 and PI3K was significant greater in rTMS group as indicated by a higher phosphorylated ERK (pY-ERK, average increase: $50 \%$ ) (Fig. 1c) and Akt ( $\mathrm{pS}^{473}$-akt, average increase: $75 \%$ ) (Fig. 1c).

BDNF-TrkB activation regulates synaptic plasticity such as LTP. This is thought to be mediated through an interaction with NMDARs. We therefore measured the levels of the obligatory NMDAR subunit, NR1; the regulatory subunit, NR2A; and the postsynaptic NMDAR anchoring protein, PSD-95, that coimmunoprecipitated with TrkB. BDNF stimulation increased the association of TrkB with NMDAR in both groups. Specifically, in the anti-TrkB immunoprecipitate of sham-treated rats, BDNF elic-

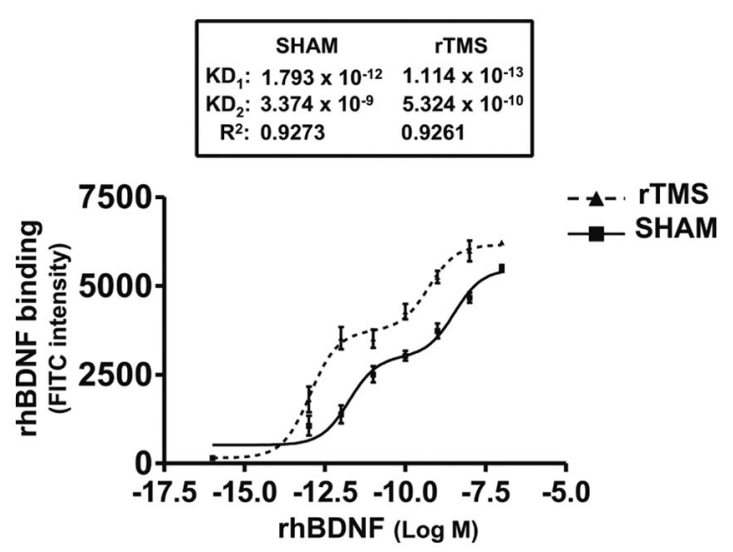

Figure 4. rTMS treatments increase BDNF affinity to TrkB in prefrontal cortex. The effect of $5 \mathrm{~d}$ rTMS on the interaction of BDNF with TrkB was assessed by a ligand binding assay. The membranebound proteins were first biotinylated using a biotinylation kit. Following detergent solubilization and then dilution, the biotinylated surface proteins were coated onto streptavidin-coated plates (ReactiBind NeutrAvidin high binding capacity coated 96 -well plate). Plates were washed and incubated at $30^{\circ} \mathrm{C}$ with $\mathrm{K}-\mathrm{R}$, and BDNF (100 fM to $10 \mathrm{~nm}$ ) was added for $1 \mathrm{~h}$. The plate was washed and then sequentially incubated with anti-BDNF followed by FITC-conjugated anti-rabbit lgG. Plates were washed and the residual FITC signals were determined by a multimode plate reader, DTX880 (Beckman). Nonlinear regression data curve fit was performed using Prism. Data points are means and vertical bars are the SEM derived from six independent rats in each treatment group.

ited a fourfold to fivefold increase of NR1 and NR2A subunits (Fig. 2a,b). The increases of NR1 and NR2A levels in the antiTrkB immunoprecipitates of rTMS-treated rats were significantly higher than in sham-treated animals (average increase: 

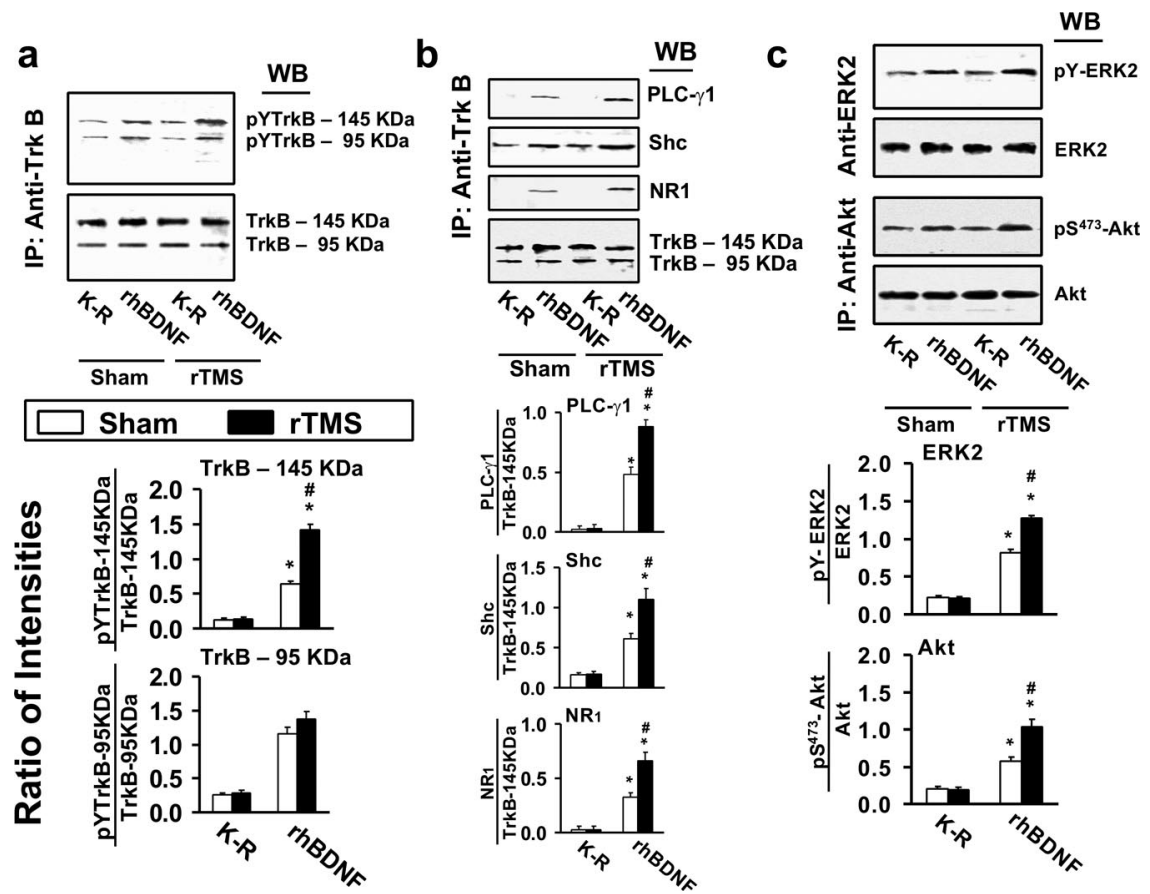

Figure 5. rTMS treatments increase BDNF-TrkB signaling and TrkB-NMDAR interaction in lymphocytes. $\boldsymbol{a}-\boldsymbol{c}$, Representative blots (top) with normalized densitometric data (bottom) showing the Western analysis of the effect of $5 \mathrm{~d} r T M S$ on tyrosine-phosphorylated (pY) TrkB levels $(\boldsymbol{a})$; the levels of PLC $\gamma 1$, adaptor protein shc, and NMDAR-NR1 subunit recruited to TrkB $(\boldsymbol{b})$; and the levels of activated ERK2 (pY-ERK2) and phosphorylated Akt (pS473-Akt) (c) in response to exogenously added rhBDNF in the anti-TrkB $(\boldsymbol{a}, \boldsymbol{b})$ and -ERK2 or Akt (c) immunoprecipitates of lymphocyte lysates prepared from sham-treated or $5 \mathrm{~d}$ rTMS rats. The blots were stripped and reprobed with anti-TrkB $(\boldsymbol{a}, \boldsymbol{b})$ and -ERK2 or Akt (c) to measure immunoprecipitation efficiency and loading. The densitometric quantification was done on six sham control/rTMS pairs. Data are means \pm SEM of the ratio of the optical intensity of the pY-TrkB $(\boldsymbol{a})$ or PLC $\gamma 1$, Shc, or NR1 (b) to the TrkBbandorthepY-ERK2 (c) andpS473-Akt(c) to theERK2and Akt, respectively, derived from sixindependentdeterminations. ${ }^{*} p<0.01$ compared to respective Krebs'-Ringer-treated level in the same group. ${ }^{\#} p<0.01$ compared to respective response in the sham control group.
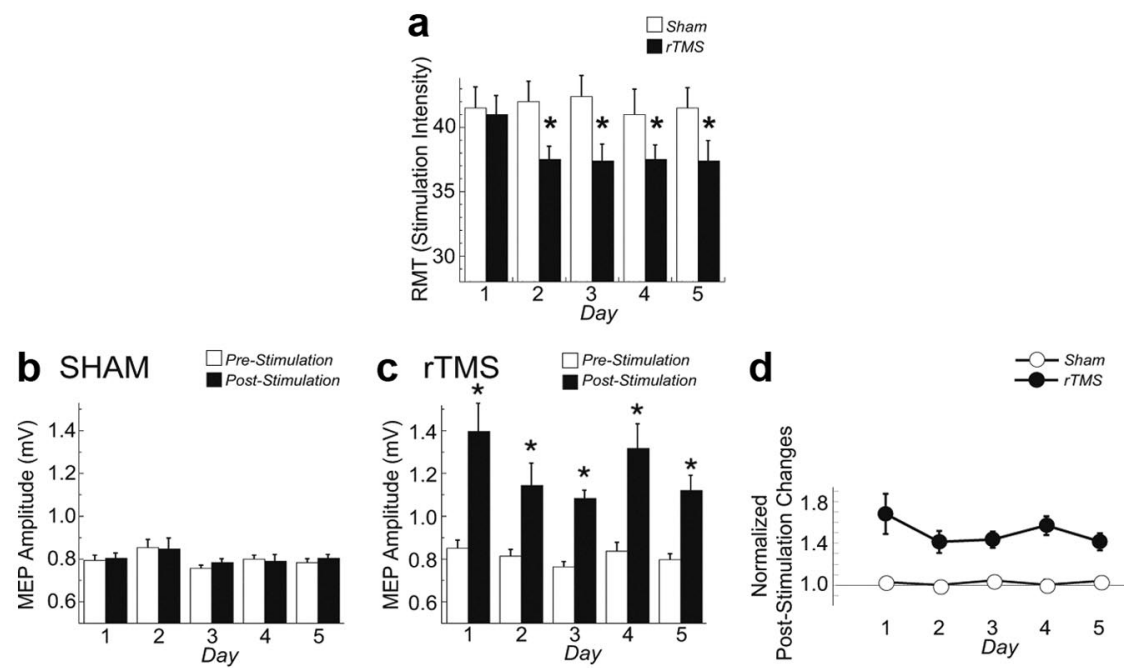

Figure 6. The effects of rTMS on RMT and on the amplitude of MEP. $\boldsymbol{a}$, RMT is plotted as a function of day for the rTMS and sham treatment. RMT significantly decreased in the rTMS but not in the sham session starting from the second day of treatment. The asterisk indicates significant differences $(p<0.005)$ with a post hoc test. $\boldsymbol{b}, \boldsymbol{c}$, Mean MEP amplitude before and immediately after sham $(\boldsymbol{b})$ and rTMS $(\boldsymbol{c})$ are plotted as a function of day. MEP amplitude significantly increased only after each rTMS treatment (asterisks: $p<0.0001$ ).d, The poststimulation changes (computed as the pre-post stimulation MEP differences normalized by the pre-MEP amplitude for each day) did not differ across days $(p>0.1)$ for either sham or rTMS treatment.

80\%) (Fig. 2a,b). In addition, BDNF stimulation induced a fourfold increase in the PSD-95 level in the sham group (Fig. 2a,b), whereas in the rTMS group the increase in TrkB-associated PSD-95 was 100\% over that of the sham group (Fig. 2a,b). Col- lectively, these data indicate that a $5 \mathrm{~d}$ rTMS treatment improves the efficacy of BDNF-TrkB signaling and TrkBNMDAR interaction in the rat PFCX.

As in the PFCX, the levels of both 145 and $95 \mathrm{kDa}$ forms of TrkB in hippocampal slices were unaffected by rTMS when measure in the presence or absence of BDNF. In the sham-treated animals, BDNF evoked a 7.8- to 9.8-fold increase in pY-145 kDa TrkB and a 4.9 - to 5.9 -fold increase in $\mathrm{pY}-95 \mathrm{kDa}$ TrkB (Table 2). In contrast to PFCX, rTMS did not significantly affect BDNF-induced pY-145 kDa or pY-95 kDa TrkB levels ( $p=$ 0.28 ) (Table 2). These effects were corroborated by the increased levels of shc and PLC- $\gamma 1$ recruited to the pY-TrkB. PLC- $\gamma 1$, shc, and $\mathrm{N}$-shc levels were comparable in the rTMS-treated and sham-treated animals ( $p=0.32$ ) (Table 2). Further support of the BDNF-induced TrkB activation was drawn from activation of the signaling cascades downstream of $\mathrm{TrkB}$ activation. In hippocampus, BDNF-induced ERK2 and PI3K activation as indicated by the increases in phosphorylated ERK (pY-ERK) and akt $\left(\mathrm{pS}^{473}\right.$-akt) was again similar in rTMS- and sham-treated rats $(p=0.44$ for $p Y-E R K$ and $p=0.35$ for $\mathrm{pS}^{473} \mathrm{Akt}$ ) (Table 2). In both PFCX and hippocampus, rTMS did not alter the expression levels of 145 or 95 $\mathrm{kDa}$ TrkB, pro-BDNF (32 kDa), or BDNF $(14 \mathrm{kDa})$ (Table 2). In keeping with the regulatory role of TrkB in NMDAR-dependent synaptic plasticity, BDNF stimulation also increased the level of TrkB-NMDAR association in the hippocampus. This is supported by the data showing that BDNF increased NR1 (4.1- to 5.5-fold) and NR2A (3.6- to 4.6-fold) subunits in the anti-TrkB immunoprecipitate derived from the hippocampal slices of sham-treated rats (Table 2). In addition, BDNF stimulation also induced a 3.6- to 4.5 -fold increase in the abundance of PSD-95. The BDNF-induced increase in TrkB-associated NMDARs and PSD-95 in hippocampus was, however, not affected by the $5 \mathrm{~d}$ rTMS treatment ( $p=$ 0.17) (Table 2). Thus, these data indicate that $5 \mathrm{~d}$ rTMS treatment improves the efficacy of BDNF-TrkB signaling in PFCX but not in the hippocampus, although there are no discernible changes in the expression of 145 and $95 \mathrm{kDa}$ TrkB (Fig. 1a), pro-BDNF $(32 \mathrm{~K}-\mathrm{Da})$, and BDNF $(14 \mathrm{kDa})$ in both brain regions (Fig. 3a).

One reason for this discrepancy could be that TMS reached only the prefrontal areas and not the hippocampal structures because PFCX is more superficial. However, the short distance (1 $\mathrm{cm}$ ) between the two and the high stimulation intensity used $(50 \%)$ makes it unlikely that the hippocampus was not reached by TMS. Alternatively, the lack of effect at the hippocampal level 
could be due to the fact that TrkB activation in this area may saturate faster and also decrease faster than in the other areas, so that $24 \mathrm{~h}$ after the last stimulation when the animals were killed, no detectable effect remained.

\section{Improved BDNF binding affinity is responsible for rTMS-induced increase in TrkB signaling in PFCX}

The increased BDNF-induced TrkB activation following rTMS in PFCX could have resulted from altered BDNF binding affinity, since no discernible changes in TrkB level were found. We thus used a modified binding assay with biotinylated surface receptors as the tissue source and fluorescence detection. We found that the saturation curves of the BDNF in PFCX from both sham- and rTMS-treated rats fit significantly better when the nonlinear regression curve-fit algorithm assumed the presence of two saturation sites rather than one $\left(r^{2}>0.92\right)$. The calculated $K_{\mathrm{d}}$ values for the sham-treated brains were $1.79 \mathrm{pm}$ and $3.37 \mathrm{~nm}$ (Fig. 4). As shown in Figure 4, in the rTMS-treated animals, BDNF binding affinities significantly increased by $\sim 10$-fold, to $0.11 \mathrm{pM}$ and 0.53 nM. These data strongly suggest that the improved BDNF binding affinity is responsible for rTMS-induced BDNF-TrkB signaling increase.

\section{rTMS increases BDNF-TrkB signaling and TrkB-NMDAR association in lymphocytes}

To determine whether rTMS also affected BDNF-TrkB signaling and their interaction with the NMDARs in peripheral tissues, we measured BDNF-induced TrkB activation in isolated lymphocytes. The results of the statistical analyses are reported in Table 1 (Lymphocytes).

BDNF-induced TrkB activation was detected in lymphocytes from both sham- and rTMS-treated rats, as indicated by the increased pY-TrkB levels. As in brain slices, BDNF stimulation did not affect the levels of both 145 and $95 \mathrm{kDa}$ forms of TrkB in lymphocytes from both animal groups (Fig. $5 a$ ). BDNF increased the levels of pY-TrkB 145 and $95 \mathrm{kDa}$ in the lymphocytes of all animals, although both were barely detectable in Krebs'-Ringer (K-R)-exposed lymphocytes (Fig. 5a). Interestingly, the levels of BDNF-induced pY-TrkB $145 \mathrm{kDa}$ were significantly greater (by $\sim 120 \%$ ) in the lymphocytes from rTMS-treated rats than in those of the sham-treated group. However, neither pY-TrkB 95 $\mathrm{kDa}$ levels (Fig. $5 a$ ) nor TrkB expression levels (Fig. $3 b$ ) in lymphocytes were affected by rTMS treatment.

In contrast with the finding that rTMS did not affect BDNF level in the PFCX, hippocampus, and CSF (for CSF, see Fig. $3 c$ ), plasma BDNF levels in the rTMS group increased by almost threefold compared to the sham group, without evidence of proBDNF (Fig. 3d). Interestingly, in the lymphocytes of all animals, we did not find detectable levels of either pro-BDNF or BDNF.

The greater BDNF-induced TrkB activation in the lymphocytes of the rTMS group compared to the sham group was also reflected by the significantly higher levels of BDNF-induced pY-
Table 3. Pearson $r$ coefficients for BDNF-TrkB signaling correlations in rat and human experiments

\begin{tabular}{llll}
\hline & NMDA/Gly & $\mathrm{K}^{+}$ & rhBDNF \\
\hline BDNF-TrkB signaling in lymphocytes and PFCX in rats & & & \\
pY-TrkB (145 kDa) & $\mathbf{0 . 6 6}$ & $\mathbf{0 . 7 4}$ & 0.55 \\
PLC- $\gamma 1$ & $\mathbf{0 . 6 4}$ & $\mathbf{0 . 6 7}$ & $\mathbf{0 . 7 2}$ \\
Shc & $\mathbf{0 . 6 6}$ & $\mathbf{0 . 5 9}$ & $\mathbf{0 . 6 9}$ \\
BDNF-TrkB signaling in lymphocytes and RMT changes & & & \\
$\quad$ in humans & & & \\
pY-TrkB (145 kDa) & $\mathbf{0 . 7 3}$ & & \\
Shc & $\mathbf{0 . 6 9}$ & & \\
pY-ERK2 & $\mathbf{0 . 7 6}$ & & \\
BDNF/albumin & $\mathbf{0 . 8 2}$ & & \\
\hline
\end{tabular}

Bold indicates significant results $(p<0.05)$.

TrkB, Shc, and PLC- $\gamma 1$ recruited (Fig. $5 b$ ), as well as of phosphorylated ERK (pY-ERK) and Akt (pS473-akt) (Fig. 5c). Similarly to the findings in PFCX, NR1 subunit level in the anti-TrkB immunoprecipitate was significantly elevated from nearly undetectable in K-R-incubated lymphocytic extract (Fig. 5b). In addition, the rTMS group showed a significantly higher TrkB-NMDAR association in lymphocytes than the sham-treated animals (average: 100\%) (Fig. 5).

To verify whether the results in lymphocytes predict those in the PFCX, we determined whether the levels of BDNF-TrkB signaling components and TrkB-NMDAR interaction in the lymphocytes are correlated with their counterparts in PFCX of the two animal groups. The results summarized in Table 1 (PFCX) indicate that BDNF-induced pY-TrkB (145 kDa), TrkBassociated PLC- $\gamma 1$, and Shc levels in lymphocytes significantly predicted the corresponding levels in PFCX. 
a
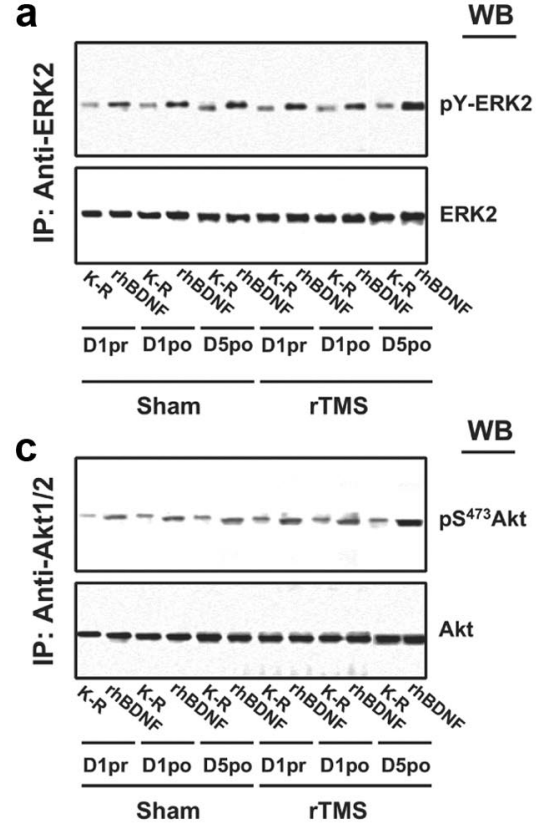

Sham

rTMS b

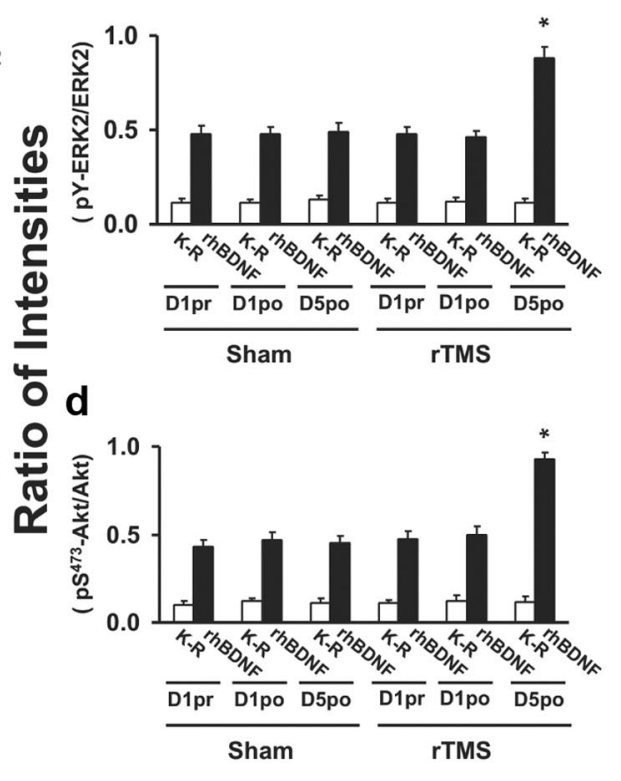

Figure 8. rTMS treatments increase TrkB downstream ERK2 and Akt activation in lymphocytes from healthy human subjects. A representative blot $(\boldsymbol{a}, \boldsymbol{b})$ with normalized densitometric data $(\boldsymbol{c}, \boldsymbol{d})$ showing the Western analysis of the effect of rTMS on the levels of pY-ERK2 and pS473-Akt in response to exogenously added rhBDNF in the anti-ERK2 and -Akt immunoprecipitates of lymphocyte lysates. Lymphocytes were prepared from blood drawn from human volunteers $1 \mathrm{~d}$ prior to (1pr) or after (1po) and $5 \mathrm{~d}$ after (5po) sham/rTMS treatments. The blots were stripped and reprobed with anti-ERK2 or -Akt to measure immunoprecipitation efficiency and loading. The densitometric quantification was done on five subjects who completed all sham and rTMS sessions. Data are means \pm SEM of ratio of the optical intensity of the pY-ERK2 and pS473-Akt to the ERK2 and Akt band, respectively. ${ }^{*} p<0.01$ compared to respective response in the sham control group.

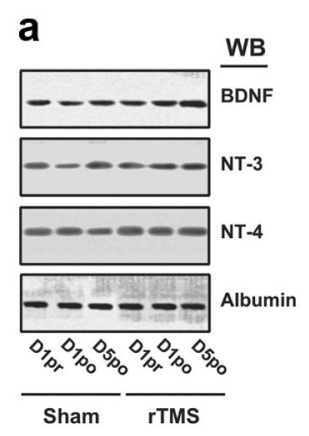

b

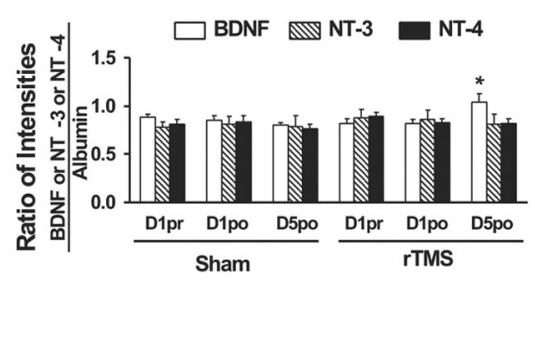

Figure 9. The effect of rTMS treatments on the expression levels of BDNF, NT-3, and NT-4 in sera from healthy human subjects. $\boldsymbol{a}$. A representative blot showing the Western analysis of the effect of sham and rTMS treatments on BDNF, NT-3, NT-4, and albumin levels in sera prepared from blood drawn from human volunteers on $1 \mathrm{~d}$ prior to ( $1 \mathrm{pr}$ ) or after ( $1 \mathrm{po}$ ) and $5 \mathrm{~d}$ after ( $5 \mathrm{po}$ ) treatments. $\boldsymbol{b}$. Normalized densitometric quantification data with albumin showing the effect of sham and rTMS treatments on BDNF, NT-3, and NT-4 levels in sera. The densitometric quantification was done on five subjects who completed all sham and rTMS sessions. Data are means \pm SEM of the ratio of the optical intensities of the BDNF, NT-3, or NT-4 to the albumin band. ${ }^{*} p<0.01$ versus respective level in each of three conditions $(1 p r, 1 p o$, or $5 p o)$ in the sham control group by a two-tailed Student's $t$ test.

Altogether, these data suggest that $5 \mathrm{~d}$ rTMS treatment improves the efficacy of BDNF-TrkB signaling in lymphocytes. The significant correlation between the levels of BDNF-TrkB signaling in lymphocytes and PFCX further suggests that TrkB function in lymphocytes reflects changes in the brain cortex.

rTMS increases BDNF-TrkB signaling and TrkB-NMDAR association in human lymphocytes

The second set of experiments was performed in humans to ascertain whether 5 consecutive days of rTMS treatment affected
BDNF-TrkB signaling and TrkBNMDAR interaction in lymphocytes. In addition, we measured changes of electrophysiological markers of cortical activity and determined whether they were correlated with changes in BDNF-TrkB activity in the lymphocytes. No subject experienced adverse effects of the rTMS procedure. Multiple rTMS, but not sham, sessions induced a significant decrease of RMT (treatment: $F_{(1,56)}=3.3, p=0.9$; day: $F_{(4,56)}=2.7, p=0.04$; treatment $\times$ day: $F_{(1,4)}=3.45, p=0.01$ ) (Fig. $6 a$ ). This decrement was already evident on the second day and was maintained throughout the entire rTMS treatment ( post hoc: $p<$ 0.005) (Fig. 6a). The RMT changes at the end of the rTMS session $(8.80 \pm 2.20 \%)$ were significantly greater than those at the end of the sham session $(-0.15 \pm 1.77 \%$; $\left.F_{(1,14)}=21.3, p=0.0004\right)$. The mean MEP amplitude did not change after each sham stimulation but, as previously shown (Quartarone et al., 2006), it increased after each $5 \mathrm{~Hz}$ rTMS session (time: $F_{(1,112)}=$ $33.8, p<0.0001$; treatment: $F_{(1,112)}=40.03$, $p<0.0001$, time $\times$ treatment: $F_{(1,1)}=$ $30.94, p<0.0001)$ (Fig. $6 b-d)$. This increase was similar over all $5 \mathrm{~d}$ (time: $F_{(4,28)}=1.07$, $p=0.39$ ) (Fig. $6 c, d)$ and did not significantly differ on day 5 compared to day 1 ( post hoc: $p=0.12$ ) (Fig. $6 d$ ).

To evaluate the acute effects of rTMS stimulation on BDNFTrkB signaling in peripheral lymphocytes, three blood samples were collected: on the first day of each session-before and $1 \mathrm{~h}$ after the stimulation - to verify the presence of acute effects, and on the fifth day-after the last stimulation-to assess chronic effects. As in the animal study, BDNF-induced TrkB activation was detected in lymphocytes from both sham and rTMS sessions, as indicated by the 5- to 10 -fold increase in pY-TrkB 145 and 95 $\mathrm{kDa}$ levels. The levels of both 145 and $95 \mathrm{kDa}$ TrkB in lymphocytes were similar with or without BDNF stimulation, without differences between the rTMS and sham sessions (Fig. 7a,b). Significant changes were found only following a $5 \mathrm{~d}$ treatment and not after a single rTMS session (for statistical analysis details, see Table 3). Specifically, after a $5 \mathrm{~d}$ rTMS treatment, the abundance of BDNF-induced pY-TrkB $145 \mathrm{kDa}$ was significantly elevated in the rTMS compared to the sham session (Fig. $7 a-c$ ). This increased level was accompanied by significant increases in TrkBassociated Shc and PLC- $\gamma 1$ (Fig. $7 b, c)$ and in activated (pY)-ERK and Akt (pS ${ }^{473}$-Akt) (Fig. 8) in response to BDNF stimulation. Moreover, compared to sham treatment, BDNF-induced TrkBNMDAR interaction increased, as indicated by significant increases of NR1 subunit level in the anti-TrkB immunoprecipitate (Fig. $7 c$ ). Similar to the findings made in rat study, $5 \mathrm{~d}$ rTMS treatment increased the plasma BDNF levels by $30 \%$ compared to the sham and $1 \mathrm{~d}$ rTMS, without evidence of pro-BDNF (Fig. 9).

We then determined whether the electrophysiological changes predicted the increased BDNF-TrkB signaling and TrkB-NMDAR interaction in the lymphocytes. Indeed, we found that the RMT changes from day 1 to day 5 significantly correlated with the corresponding changes in pY-TRKB 145, Shc, NR1, pSAkt2, and BDNF levels [Tables 2 (rTMS) and 4]. In contrast, 
Table 4. Results of ANOVA for BDNF-TrkB signaling and TrkB-NMDAR linkage comparing sham and rTMS treatments in humans

\begin{tabular}{|c|c|c|c|c|c|c|c|}
\hline & Treatme & Iam) & Time (pr & ost5) & Treatme & & rTMS versus Sham: post5 \\
\hline & $F_{(1,24)}$ & $p$ & $F_{(2,24)}$ & $p$ & $F_{(1,2)}$ & $p$ & $p$ \\
\hline pY-TRKB(145 kDa) & 21.700 & $<0.0001$ & 9.704 & 0.0008 & 12.409 & 0.0002 & 0.0028 \\
\hline pY-TRKB (95 kDa) & 2.592 & 0.1205 & 0.118 & 0.8894 & 0.345 & 0.7118 & - \\
\hline PLC $-\gamma 1$ & 6.691 & 0.0162 & 1.628 & 0.2172 & 4.580 & 0.0207 & 0.0250 \\
\hline Sch & 4.134 & 0.0532 & 7.289 & 0.0034 & 5.733 & 0.0092 & 0.0078 \\
\hline NR1 & 15.941 & 0.0005 & 2.259 & 0.1262 & 12.245 & 0.0002 & 0.0015 \\
\hline pY-ERK2 & 16.704 & 0.0004 & 13.723 & 0.0001 & 23.185 & $<0.0001$ & 0.0009 \\
\hline pS-AKT & 25.607 & $<0.0001$ & 23.995 & $<0.0001$ & 20.151 & $<0.0001$ & 0.0005 \\
\hline
\end{tabular}

Bold indicates significant results.

there was no correlation between the changes in MEP amplitude and BDNF-TrkB signaling in lymphocytes.

\section{Discussion}

The results of our animal work show that multiple rTMS sessions uplift BDNF-TrkB signaling and TrkB-NMDAR interaction, not just in PFCX, but also in lymphocytes. Importantly, the magnitudes of such activations in the two sites were significantly correlated. In humans, we found that a $5 \mathrm{~d} 5 \mathrm{~Hz}$ rTMS protocol similarly increased BDNF-induced TrkB activation in lymphocytes. Importantly, this increase correlated with the RMT decrement induced by rTMS treatment.

\section{rTMS increases BDNF-TrkB signaling in PFCX of rats}

This is the first direct demonstration that rTMS produces cortical plastic changes by promoting BDNF-TrkB signaling and TrkBNMDAR interaction. Similar findings have been reported in animals using transcranial direct current stimulation (Fritsch et al., 2010). BDNF-TrkB signaling regulates multiple brain functions, from differentiation and neuronal survival to synaptogenesis and activity-dependent forms of synaptic plasticity (Lu et al., 2008). Particularly relevant is the previous finding that BDNF-TrkB signaling mediates synaptic plasticity-related learning and memory with fear learning and extinction paradigms (Soliman et al., 2010). Thus, it is conceivable that $5 \mathrm{~d}$ rTMS treatment increases synaptic efficacy to promote LTP, a cellular correlate of learning and memory (Esser et al., 2006; Quartarone et al., 2006; Di Lazzaro et al., 2010). The effects of rTMS on BDNF-TrkB signaling are likely on both presynaptic and postsynaptic compartments, since both $\mathrm{K}^{+}$-depolarization and NMDA/glycine stimulation lead to a significantly higher levels of activated (pY) $145 \mathrm{kDa}$ TrkB but not of the dominant-negative, truncated $95 \mathrm{kDa}$ TrkB (Eide et al., 1996). Since the expression of BDNF and TrkB were not altered, the improved BDNF-TrkB signaling must be the result of increased TrkB affinity for BDNF. Indeed, the combination of increased sensitivity of TrkB for BDNF and sustained, robust TrkB-NMDAR interaction leads to higher and more persistent NMDAR-dependent LTP (Figurov et al., 1996), as well as to synaptogenesis (Elmariah et al., 2005). These events ultimately result in a protracted, if not permanent, upregulation of synaptic excitability, as BDNF-induced TrkB activation enhances glutamatergic neurotransmission (Levine et al., 1995), rapid NMDAR subunit phosphorylation (Suen et al., 1997), and LTP (Figurov et al., 1996).

\section{rTMS increases cortical excitability in humans}

Our study in humans revealed that, in agreement with previous studies (Quartarone et al., 2005, 2006), subthreshold 5 Hz rTMS over the motor cortex induced acutely an increase in the MEP amplitude and, over the course of a few treatments, a reduction of RMT. RMT is the minimum intensity that produces MEPs in the target muscle on 50\% of trials (Rothwell et al., 1999). The threshold for producing an MEP in resting muscle reflects the excitability of a central core of neurons, located in the proximity of the stimulating coil. As it can be influenced by drugs affecting sodium and calcium channels, RMT value must indicate membrane excitability (Hallett, 2000) and, thus, could probably reflect mechanisms other than synaptic changes. On the other hand, turning up stimulus intensity increases MEP size as a function of stimulus strength. The bases of MEP amplitude are less well understood, but they must involve neurons other than those in the core region activated at threshold. These neurons have higher threshold for activation, either because they are intrinsically less excitable or because they are further from the center of activation by the magnetic stimulus. In addition, at intensity above $80 \%$, TMS induce a descending volley that produces remote spinal effects. Thus, at higher intensity, TMS aftereffects become very complex and may reflect excitability changes of both cortical, subcortical (Strafella et al., 2003) and spinal synaptic relays. For these reasons, it is not surprising that only the RMT changes, which are purely cortical in nature, significantly correlated with the corresponding changes in pY-TRKB 145, Shc, NR1, pSAkt2, and BDNF levels at lymphocyte level. The complex TMS interaction within the motor system at higher intensities makes correlation with NMDA transmission extremely unlikely.

\section{rTMS increases BDNF levels in the plasma}

One of the novel findings is that multiple rTMS treatments elevate the BDNF levels in the plasma of both rats and humans, in agreement with previous reports of increased BDNF levels in the serum of depressed patients following multiple rTMS treatments (Yukimasa et al., 2006; Zanardini et al., 2006). Interestingly, the results of our animal study suggest that the sources of BDNF increase may likely reside outside the CNS, since both rTMS- and sham-treated rats had similar BDNF levels in the corticospinal fluid and the brain. BDNF increases could have resulted from the activation of muscles during the last rTMS treatment. However, this is unlikely, as the increase of plasma BDNF in humans following exercise seems to be independent from that produced by muscles (Matthews et al., 2009). Hence, the origin of increased plasma BDNF following rTMS remains elusive. Nevertheless, our findings that rTMS improves BDNF-TrkB signaling and increases plasma BDNF level support the application of rTMS for the treatment of depression. In fact, in depression and anxiety disorder with impaired extinction learning and hypofunctional PFCX, lower plasma BDNF levels have been associated with the reduced BDNF-TrkB signaling (Martinowich et al., 2007; Soliman et al., 2010).

\section{rTMS increases BDNF-TrkB signaling in lymphocytes}

The most intriguing finding of this study is that the effects of rTMS in both rats and humans extended beyond the CNS. In fact, 
following $5 \mathrm{~d}$ rTMS treatment, BDNF-TrkB signaling and TrkBNMDAR interaction increased in the lymphocytes. Together with the fact that rTMS increased plasma BDNF but not NT3 or NT4 levels (Fig. 9), the data from the studies in rats strongly suggest that rTMS may selectively upregulate the efficacy of BDNF-TrkB signaling by uplifting the TrkB sensitivity for $\mathrm{BDNF}$ and/or increasing BDNF levels. Importantly, the increased BDNF-TrkB signaling, but not TrkB-NMDAR interaction in lymphocytes, was significantly correlated with those in PFCX. These findings are further supported by the results of the human studies, in which we found correlations between changes in RMT and BDNF-TrkB signaling, but not TrkBNMDAR interaction. Altogether, the human and animal results suggest that the enhanced BDNF-TrkB signaling in lymphocytes parallels BDNF-TrkB cortical activity.

It is well documented that TrkB are present in the lymphocytes (Schuhmann et al., 2005; De Santi et al., 2009), although their function in these cells has not been thoroughly elucidated. Through TrkB activation, BDNF promotes maturation of the immune system as well as development, maintenance, and survival of lymphocytes (Schuhmann et al., 2005). The link between the immune system and the brain does not come as a surprise, as numerous studies have now shown that the two systems can interact bidirectionally, through common receptors and ligands, such as interleukin- $1 \beta$ and other proinflammatory cytokines (Derecki et al., 2010; Yirmiya and Goshen, 2011). The results of the present study further suggest that the induction of plasticityrelated phenomena in the brain also has an effect on the immune system. This conclusion is in agreement with studies showing that young mice receiving lymphocytes from senescent mice decreased their learning abilities to the level of senescent mice and showed senescence-like serum-brain reactivity (Lal et al., 1986). Moreover, there is evidence that immune processes may be involved in the pathogenesis of the neurological disorders with altered synaptic plasticity, such as Alzheimer's disease (Zotova et al., 2010).

The precise functional meaning of a parallel BDNF-TrkB signaling in cortex and lymphocytes remains speculative at the present time, since a causal link between the two phenomena has yet to be established. Nevertheless, given the correlation between BDNF-TrkB signaling in lymphocytes and PFCX in rats and with RMT changes in humans, it is plausible that TrkB function in lymphocytes might represent a promising "in vivo" marker to predict changes of that activity in cortical regions.

\section{Relevance of these findings to neuropsychiatric disorders}

There is increasing evidence that NMDA-dependent cortical plasticity is altered in many neuropsychiatric illnesses (Hardingham and Bading, 2010), including Alzheimer disease (Wang et al., 2009), Parkinson's disease (Meoni et al., 1999), dystonia (Akopian et al., 2008), and amyotrophic lateral sclerosis (Gredal et al., 1996). However, reliable and accessible peripheral markers of brain plasticity are still lacking. The results of our study suggest that BDNF-TrkB signaling is involved in cortical synaptic plasticity and that such activity might be reflected in lymphocytes. Ad hoc-designed studies are now needed to determine a causal relationship between the two findings and, thus, to ascertain whether TrkB activity in the lymphocytes can be used as a reliable predictor of cortical synaptic plasticity. Indeed, elucidating the cellular and molecular bases of the effects of TMS on brain and other tissues will be invaluable for characterizing neurological dysfunctions and for promoting effective treatments in diverse neurological and psychiatric disorders.

\section{References}

Akopian G, Crawford C, Beal MF, Cappelletti M, Jakowec MW, Petzinger GM, Zheng L, Gheorghe SL, Reichel CM, Chow R, Walsh JP (2008) Decreased striatal dopamine release underlies increased expression of long-term synaptic potentiation at corticostriatal synapses $24 \mathrm{~h}$ after 3-nitropropionic-acidinduced chemical hypoxia. J Neurosci 28:9585-9597.

Berzi A, Ayata CK, Cavalcante P, Falcone C, Candiago E, Motta T, Bernasconi P, Hohlfeld R, Mantegazza R, Meinl E, Farina C (2008) BDNF and its receptors in human myasthenic thymus: implications for cell fate in thymic pathology. J Neuroimmunol 197:128-139.

Conte A, Belvisi D, Iezzi E, Mari F, Inghilleri M, Berardelli A (2008) Effects of attention on inhibitory and facilitatory phenomena elicited by pairedpulse transcranial magnetic stimulation in healthy subjects. Exp Brain Res 186:393-399.

Derecki NC, Cardani AN, Yang CH, Quinnies KM, Crihfield A, Lynch KR, Kipnis J (2010) Regulation of learning and memory by meningeal immunity: a key role for IL-4. J Exp Med 207:1067-1080.

De Santi L, Cantalupo L, Tassi M, Raspadori D, Cioni C, Annunziata P (2009) Higher expression of BDNF receptor gp 145 trkB is associated with lower apoptosis intensity in T cell lines in multiple sclerosis. J Neurol Sci 277:65-70.

Di Lazzaro V, Profice P, Pilato F, Dileone M, Oliviero A, Ziemann U (2010) The effects of motor cortex rTMS on corticospinal descending activity. Clin Neurophysiol 121:464-473.

Dissen GA, Hirshfield AN, Malamed S, Ojeda SR (1995) Expression of neurotrophins and their receptors in the mammalian ovary is developmentally regulated: changes at the time of folliculogenesis. Endocrinology 136:4681-4692.

Easton JB, Moody NM, Zhu X, Middlemas DS (1999) Brain-derived neurotrophic factor induces phosphorylation of fibroblast growth factor receptor substrate 2. J Biol Chem 274:11321-11327.

Eide FF, Vining ER, Eide BL, Zang K, Wang XY, Reichardt LF (1996) Naturally occurring truncated trkB receptors have dominant inhibitory effects on brain-derived neurotrophic factor signaling. J Neurosci 16:3123-3129.

Elahi B, Elahi B, Chen R (2009) Effect of transcranial magnetic stimulation on Parkinson motor function-systematic review of controlled clinical trials. Mov Disord 24:357-363.

Elmariah SB, Hughes EG, Oh EJ, Balice-Gordon RJ (2005) Neurotrophin signaling among neurons and glia during formation of tripartite synapses. Neuron Glia Biol 1:1-11.

Esser SK, Huber R, Massimini M, Peterson MJ, Ferrarelli F, Tononi G (2006) A direct demonstration of cortical LTP in humans: a combined TMS/EEG study. Brain Res Bull 69:86-94.

Figurov A, Pozzo-Miller LD, Olafsson P, Wang T, Lu B (1996) Regulation of synaptic responses to high-frequency stimulation and LTP by neurotrophins in the hippocampus. Nature 381:706-709.

Fregni F, Pascual-Leone A (2007) Technology insight: noninvasive brain stimulation in neurology-perspectives on the therapeutic potential of rTMS and tDCS. Nat Clin Pract Neurol 3:383-393.

Fritsch B, Reis J, Martinowich K, Schambra HM, Ji Y, Cohen LG, Lu B (2010) Direct current stimulation promotes BDNF-dependent synaptic plasticity: potential implications for motor learning. Neuron 66:198-204.

Gredal O, Witt MR, Dekermendjian K, Møller SE, Nielsen M (1996) Cerebrospinal fluid from amyotrophic lateral sclerosis has no effect on intracellular free calcium in cultured cortical neurons. Mol Chem Neuropathol 29:141-152.

Greenberg ME, Xu B, Lu B, Hempstead BL (2009) New insights in the biology of BDNF synthesis and release: implications in CNS function. J Neurosci 29:12764-12767.

Hallett M (2000) Transcranial magnetic stimulation and the human brain. Nature 406:147-150.

Hardingham GE, Bading H (2010) Synaptic versus extrasynaptic NMDA receptor signalling: implications for neurodegenerative disorders. Nat Rev Neurosci 11:682-696.

Hiltunen JO, Arumäe U, Moshnyakov M, Saarma M (1996) Expression of mRNAs for neurotrophins and their receptors in developing rat heart. Circ Res 79:930-939.

Kermani P, Rafii D, Jin DK, Whitlock P, Schaffer W, Chiang A, Vincent L, Friedrich M, Shido K, Hackett NR, Crystal RG, Rafii S, Hempstead BL (2005) Neurotrophins promote revascularization by local recruitment of TrkB + endothelial cells and systemic mobilization of hematopoietic progenitors. J Clin Invest 115:653-663. 
Lal H, Bennett M, Bennett D, Forster MJ, Nandy K (1986) Learning deficits occur in young mice following transfer of immunity from senescent mice. Life Sci 39:507-512.

Levine ES, Dreyfus CF, Black IB, Plummer MR (1995) Brain-derived neurotrophic factor rapidly enhances synaptic transmission in hippocampal neurons via postsynaptic tyrosine kinase receptors. Proc Natl Acad Sci U S A 92:8074-8077.

Longo FM, Yang T, Knowles JK, Xie Y, Moore LA, Massa SM (2007) Small molecule neurotrophin receptor ligands: novel strategies for targeting Alzheimer's disease mechanisms. Curr Alzheimer Res 4:503-506.

Lu Y, Christian K, Lu B (2008) BDNF: a key regulator for protein synthesis-dependent LTP and long-term memory? Neurobiol Learn Mem 89:312-323.

Maroder M, Bellavia D, Meco D, Napolitano M, Stigliano A, Alesse E, Vacca A, Giannini G, Frati L, Gulino A, Screpanti I (1996) Expression of trKB neurotrophin receptor during $\mathrm{T}$ cell development. Role of brain derived neurotrophic factor in immature thymocyte survival. J Immunol 157:2864-2872.

Martinowich K, Manji H, Lu B (2007) New insights into BDNF function in depression and anxiety. Nat Neurosci 10:1089-1093.

Matthews VB, Aström MB, Chan MH, Bruce CR, Krabbe KS, Prelovsek O, Akerström T, Yfanti C, Broholm C, Mortensen OH, Penkowa M, Hojman P, Zankari A, Watt MJ, Bruunsgaard H, Pedersen BK, Febbraio MA (2009) Brain-derived neurotrophic factor is produced by skeletal muscle cells in response to contraction and enhances fat oxidation via activation of AMP-activated protein kinase. Diabetologia 52:1409-1418.

Meoni P, Bunnemann BH, Kingsbury AE, Trist DG, Bowery NG (1999) NMDA NR1 subunit mRNA and glutamate NMDA-sensitive binding are differentially affected in the striatum and pre-frontal cortex of Parkinson's disease patients. Neuropharmacology 38:625-633.

Minichiello L (2009) TrkB signalling pathways in LTP and learning. Nat Rev Neurosci 10:850-860.

Nirogi R, Kandikere V, Mudigonda K, Bhyrapuneni G, Muddana N, Saralaya R, Benade V (2009) A simple and rapid method to collect the cerebrospinal fluid of rats and its application for the assessment of drug penetration into the central nervous system. J Neurosci Methods 178:116-119.

Pflug BR, Dionne C, Kaplan DR, Lynch J, Djakiew D (1995) Expression of a Trk high affinity nerve growth factor receptor in the human prostate. Endocrinology 136:262-268.

Quartarone A, Bagnato S, Rizzo V, Morgante F, Sant'angelo A, Battaglia F, Messina C, Siebner HR, Girlanda P (2005) Distinct changes in cortical and spinal excitability following high-frequency repetitive TMS to the human motor cortex. Exp Brain Res 161:114-124.

Quartarone A, Rizzo V, Bagnato S, Morgante F, Sant'Angelo A, Girlanda P, Siebner HR (2006) Rapid-rate paired associative stimulation of the median nerve and motor cortex can produce long-lasting changes in motor cortical excitability in humans. J Physiol 575:657-670.

Rossi S, Hallett M, Rossini PM, Pascual-Leone A (2009) Safety, ethical considerations, and application guidelines for the use of transcranial magnetic stimulation in clinical practice and research. Clin Neurophysiol 120:2008-2039.

Rossini PM, Barker AT, Berardelli A, Caramia MD, Caruso G, Cracco RQ, Dimitrijević MR, Hallett M, Katayama Y, Lücking $\mathrm{CH}$, Maertens de Noordhout A, Marsden C, Murray N, Rothwell J, Swash M, Tomberg C (1994) Non-invasive electrical and magnetic stimulation of the brain, spinal cord and roots: basic principles and procedures for routine clinical application. Report of an IFCN committee. Electroencephalogr Clin Neurophysiol 91:79-92.
Rothwell JC, Hallett M, Berardelli A, Eisen A, Rossini P, Paulus W (1999) Magnetic stimulation: motor evoked potentials. The International Federation of Clinical Neurophysiology. Electroencephalogr Clin Neurophysiol Suppl 52:97-103.

Schuhmann B, Dietrich A, Sel S, Hahn C, Klingenspor M, Lommatzsch M, Gudermann T, Braun A, Renz H, Nockher WA (2005) A role for brainderived neurotrophic factor in B cell development. J Neuroimmunol 163:15-23.

Schultz R, Metsis M, Hökfelt T, Parvinen M, Pelto-Huikko M (2001) Expression of neurotrophin receptors in rat testis. Upregulation of TrkA mRNA with hCG treatment. Mol Cell Endocrinol 182:121-127.

Siebner HR, Peller M, Willoch F, Minoshima S, Boecker H, Auer C, Drzezga A, Conrad B, Bartenstein P (2000) Lasting cortical activation after repetitive TMS of the motor cortex: a glucose metabolic study. Neurology 54:956-963.

Siebner HR, Takano B, Peinemann A, Schwaiger M, Conrad B, Drzezga A (2001) Continuous transcranial magnetic stimulation during positron emission tomography: a suitable tool for imaging regional excitability of the human cortex. Neuroimage 14:883-890.

Soliman F, Glatt CE, Bath KG, Levita L, Jones RM, Pattwell SS, Jing D, Tottenham N, Amso D, Somerville LH, Voss HU, Glover G, Ballon DJ, Liston C, Teslovich T, Van Kempen T, Lee FS, Casey BJ (2010) A genetic variant BDNF polymorphism alters extinction learning in both mouse and human. Science 327:863-866.

Strafella AP, Paus T, Fraraccio M, Dagher A (2003) Striatal dopamine release induced by repetitive transcranial magnetic stimulation of the human motor cortex. Brain 126:2609-2615.

Suen PC, Wu K, Levine ES, Mount HT, Xu JL, Lin SY, Black IB (1997) Brain-derived neurotrophic factor rapidly enhances phosphorylation of the postsynaptic N-methyl-D-aspartate receptor subunit 1. Proc Natl Acad Sci U S A 94:8191-8195.

Vedeniapin A, Cheng L, George MS (2010) Feasibility of simultaneous cognitive behavioral therapy and left prefrontal rTMS for treatment resistant depression. Brain Stimul 3:207-210.

Wang F, Geng X, Tao HY, Cheng Y (2010) The restoration after repetitive transcranial magnetic stimulation treatment on cognitive ability of vascular dementia rats and its impacts on synaptic plasticity in hippocampal CA1 area. J Mol Neurosci 41:145-155.

Wang HY, Stucky A, Liu J, Shen C, Trocme-Thibierge C, Morain P (2009) Dissociating beta-amyloid from alpha 7 nicotinic acetylcholine receptor by a novel therapeutic agent, S 24795, normalizes alpha 7 nicotinic acetylcholine and NMDA receptor function in Alzheimer's disease brain. J Neurosci 29:10961-10973.

Yirmiya R, Goshen I (2011) Immune modulation of learning, memory, neural plasticity and neurogenesis. Brain Behav Immun 25:181-213.

Yukimasa T, Yoshimura R, Tamagawa A, Uozumi T, Shinkai K, Ueda N, Tsuji S, Nakamura J (2006) High-frequency repetitive transcranial magnetic stimulation improves refractory depression by influencing catecholamine and brain-derived neurotrophic factors. Pharmacopsychiatry 39:52-59.

Zanardini R, Gazzoli A, Ventriglia M, Perez J, Bignotti S, Rossini PM, Gennarelli M, Bocchio-Chiavetto L (2006) Effect of repetitive transcranial magnetic stimulation on serum brain derived neurotrophic factor in drug resistant depressed patients. J Affect Disord 91:83-86.

Zotova E, Nicoll JA, Kalaria R, Holmes C, Boche D (2010) Inflammation in Alzheimer's disease: relevance to pathogenesis and therapy. Alzheimers Res Ther 2:1. 